International Journal of Pure and Applied Mathematics

Volume 87 No. 2 2013, 273-276

ISSN: 1311-8080 (printed version); ISSN: 1314-3395 (on-line version)

url: http://www.ijpam.eu

doi: http://dx.doi.org/10.12732/ijpam.v87i2.7

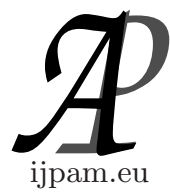

\title{
IMPROVEMENT OF POISSON APPROXIMATION FOR INDEPENDENT GEOMETRIC TRIALS
}

\author{
K. Teerapabolarn \\ Department of Mathematics \\ Faculty of Science \\ Burapha University \\ Chonburi, 20131, THAILAND
}

\begin{abstract}
We use the Stein-Chen method to improve the bound in [5] to be more appropriate for the Poisson approximation to a sum of independently distributed geometric random variables.
\end{abstract}

AMS Subject Classification: 62E17, 60F05, 60G50

Key Words: distribution function, geometric random variable, Poisson approximation, Stein-Chen method

\section{Introduction}

Let $W$ be a sum $\sum_{i=1}^{n} X_{i}$ of independently distributed geometric random variables, each with probability $P\left(X_{i}=k\right)=\left(1-p_{i}\right)^{k} p_{i}$ for $k=0,1, \ldots$ It is well-known that if all $q_{i}=\left(1-p_{i}\right)$ are small, the distribution of $W$ can be approximated by the Poisson distribution with mean $\lambda=E(W)=\sum_{i=1}^{n} q_{i} p_{i}^{-1}$. Correspondingly, the distribution function of $W$ can also be approximated by the Poisson distribution function with mean $\lambda$. Let $\mathbb{P} \mathbb{W}\left(w_{0}\right)=P\left(W \leq w_{0}\right)$ and $\mathbb{P}_{\lambda}\left(w_{0}\right)=\sum_{k=0}^{w_{0}} \frac{\lambda^{k} e^{-\lambda}}{k !}$ be the distribution function of $W$ and the Poisson distribution function at $w_{0} \in \mathbb{N} \cup\{0\}$, respectively. In this case, Teerapabolarn and Wongkasem [5] used the Stein-Chen method to give a non-uniform bound

Received: March 26, 2013

(c) 2013 Academic Publications, Ltd. url: www.acadpubl.eu 
for the difference of $\mathbb{P} \mathbb{W}\left(w_{0}\right)$ and $\mathbb{P}_{\lambda}\left(w_{0}\right)$ as follows:

$$
\left|\mathbb{P} \mathbb{W}\left(w_{0}\right)-\mathbb{P}_{\lambda}\left(w_{0}\right)\right| \leq \lambda^{-1}\left(e^{\lambda}-1\right) \sum_{i=1}^{n} \min \left\{1, \frac{1}{p_{i}\left(w_{0}+1\right)}\right\} q_{i}^{2} p_{i}^{-1},
$$

where $w_{0} \in \mathbb{N} \cup\{0\}$. We observe that the result in (1.1) gives a good Poisson approximation whenever $e^{\lambda}$ is small; however, when $e^{\lambda}$ is large, this result may be inappropriate for approximating the distribution function of $W$. In this paper, our goal is to improve the results in (1.1) by eliminating the influence of the factor $e^{\lambda}$.

\section{Method}

Stein's method was originally formulated for normal approximation by Stein [2]. It was adapted and applied to the Poisson case by Chen [1], which is refer to as the Stein-Chen method. Following Teerapabolarn [3], Stein's equation of the Poisson cumulative distribution function with parameter $\lambda>0$ is of the form

$$
h_{w_{0}}(w)-\mathbb{P}_{\lambda}\left(w_{0}\right)=\lambda f_{w_{0}}(w+1)-w f_{w_{0}}(w),
$$

where $w_{0}, w \in \mathbb{N} \cup\{0\}$, and for $h_{w_{0}}(w)=1$ if $w \leq w_{0}$ and $h_{w_{0}}(w)=0$ if $w>w_{0}$, the solution $f_{w_{0}}$ is

$$
f_{w_{0}}(w)= \begin{cases}(w-1) ! \lambda^{-w} e^{\lambda}\left[\mathbb{P}_{\lambda}(w-1)\left[1-\mathbb{P}_{\lambda}\left(w_{0}\right)\right]\right] & \text { if } w \leq w_{0} \\ (w-1) ! \lambda^{-w} e^{\lambda}\left[\mathbb{P}_{\lambda}\left(w_{0}\right)\left[1-\mathbb{P}_{\lambda}(w-1)\right]\right] & \text { if } w>w_{0} \\ 0 & \text { if } w=0\end{cases}
$$

The following lemma gives a non-uniform bound of (2.2), which is used to improve the bound in (1.1).

Lemma 2.1. For $w_{0}, k \in \mathbb{N}$, let $\Delta f_{w_{0}}(w)=f_{w_{0}}(w+1)-f_{w_{0}}(w)$. Then the following inequality holds:

$$
\sup _{w \geq k}\left|\Delta f_{w_{0}}(w)\right| \leq \min \left\{\lambda^{-1}\left(1-e^{-\lambda}\right), \frac{1}{w_{0}}, \frac{1}{k}\right\} .
$$

Proof. The first and second bounds follow from [4]. Next, we shall show that $\sup _{w \geq k}\left|\Delta f_{w_{0}}(w)\right| \leq \frac{1}{k}$. It is clear that $\sup _{w \geq k}\left|\Delta f_{w_{0}}(w)\right| \leq \frac{1}{w_{0}} \leq \frac{1}{k}$ for $k \leq w \leq w_{0}$. $w \geq k$

$$
w \geq k
$$

For $w \geq k$ and $w>w_{0}$, it follows from [4] that $\sup _{w \geq k}\left|\Delta f_{w_{0}}(w)\right| \leq \frac{1}{w} \leq \frac{1}{k}$. Therefore, $\sup _{w \geq k}\left|\Delta f_{w_{0}}(w)\right| \leq \frac{1}{k}$, hence (2.3) holds. 


\section{Result}

The following theorem presents a new non-uniform bound on $\left|\mathbb{P W}\left(w_{0}\right)-\mathbb{P}_{\lambda}\left(w_{0}\right)\right|$.

Theorem 3.1. For $w_{0} \in \mathbb{N}$, if $\lambda=\sum_{i=1}^{n} q_{i} p_{i}^{-1}$, then we have the following:

$$
\left|\mathbb{P} \mathbb{W}\left(w_{0}\right)-\mathbb{P}_{\lambda}\left(w_{0}\right)\right| \leq \sum_{i=1}^{n} \min \left\{\frac{\lambda^{-1}\left(1-e^{-\lambda}\right)}{p_{i}}, \frac{1}{p_{i} w_{0}}, 1\right\} q_{i}^{2} p_{i}^{-1}
$$

where $\mathbb{P} \mathbb{W}(0)=\prod_{i=1}^{n} p_{i}$.

Proof. Teerapabolarn and Wongkasem [5] showed that

$$
\begin{aligned}
\left|\mathbb{P} \mathbb{W}\left(w_{0}\right)-\mathbb{P}_{\lambda}\left(w_{0}\right)\right| & \leq \sum_{i=1}^{n} \sum_{k=1}^{\infty} k q_{i}^{k+1} \sup _{w \geq k}\left|\Delta f_{w_{0}}(w)\right| \\
& \leq \sum_{i=1}^{n} \sum_{k=1}^{\infty} k q_{i}^{k+1} \min \left\{\lambda^{-1}\left(1-e^{-\lambda}\right), \frac{1}{w_{0}}, \frac{1}{k}\right\} \\
& =\sum_{i=1}^{n} \min \left\{\frac{\lambda^{-1}\left(1-e^{-\lambda}\right)}{p_{i}}, \frac{1}{p_{i} w_{0}}, 1\right\} q_{i}^{2} p_{i}^{-1}
\end{aligned}
$$

Hence, the theorem is proved.

Remark. Because min $\left\{\frac{1-e^{-\lambda}}{\lambda p_{i}}, \frac{1}{p_{i} w_{0}}, 1\right\}<\frac{e^{\lambda}-1}{\lambda} \min \left\{1, \frac{1}{p_{i}\left(w_{0}+1\right)}\right\}$ for every $w_{0} \in \mathbb{N}$. Therefore, the bound in (3.1) is sharper than that in (1.1).

\section{Conclusion}

The non-uniform bound in the Theorem 3.1, which was improved by the SteinChen method, provides an appropriate criteria for measuring the accuracy of approximating the distribution function of $W$ by the Poisson distribution function with mean $\lambda=\sum_{i=1}^{n} q_{i} p_{i}^{-1}$. In addition, the influence of factor $e^{\lambda}$ in the old bound is eliminated from or reduced in the new bound. So, the bound in this study is sharper than that reported in [5].

\section{References}

[1] L.H.Y. Chen, Poisson approximation for dependent trials, Ann. Probab., 3 (1975), 534-545. 
[2] C.M. Stein, A bound for the error in normal approximation to the distribution of a sum of dependent random variables, Proc.Sixth Berkeley Sympos. Math. Statist. Probab., 3 (1972), 583-602.

[3] K. Teerapabolarn, A note on Poisson approximation for independent geometric random variables, Int. Math. Forum, 4 (2009), 531-535.

[4] K. Teerapabolarn, A non-uniform bound on Poisson approximation by $w$ functions, Int. J. Pure Appl. Math., 78 (2012), 1063-1075.

[5] K. Teerapabolarn and P. Wongkasem, Poisson approximation for independent geometric random variables, Int. Math. Forum, 2 (2007), 3211-3218. 